Historic, Archive Document

Do not assume content reflects current scientific knowledge, policies, or practices. 


\section{PLANT LIS'I}

1932-1933

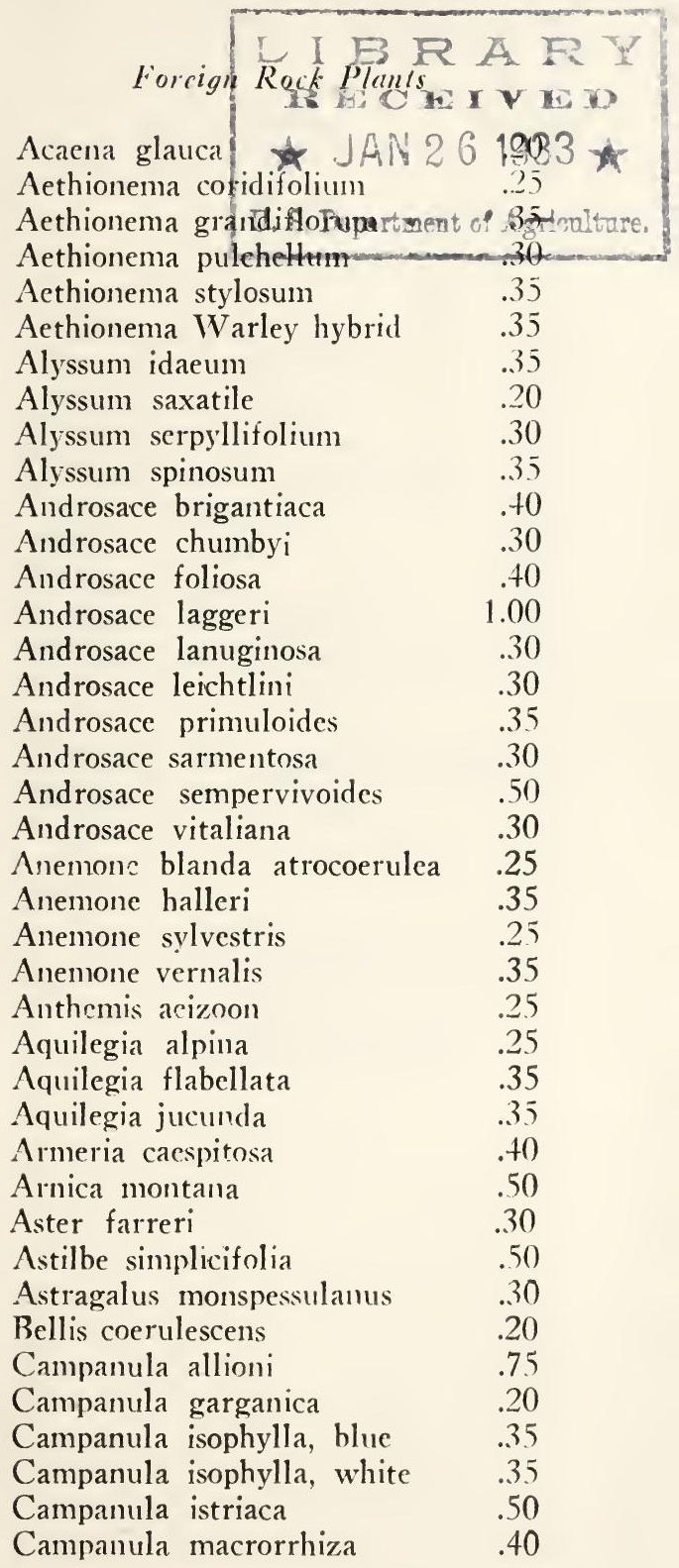


Campanula miranda $\quad .25$

Campanula miranda alba $\quad .25$

Campanula portenschlagiana $\quad .25$

Campanula pusilla $\quad .25$

Campanula pusilla alba $\quad .30$

Campanula raddeana $\quad .20$

Campanula spathulata $\quad .50$

Campanula stenocodon $\quad .50$

Campanula turbinata $\quad .50$

Commelina coelestis .4t

Delphinium tatsiense $\quad .40$

Dianthus sp. (Ljuboten) $\quad .35$

Dianthus neglectus $\quad .35$

Draba acizoon $\quad .30$

Draba cuspidata $\quad .30$

Draba pyrenaica $\quad .75$

Erinus alpinus $\quad .20$

Erodium chamaedryoides $\quad 1.00$

Erodium petraeum $\quad .50$

Epimedium sulphurcum $\quad .40$

Gentiana acaulis $\quad .75$

Gentiana excisa $\quad 1.00$

Gentiana farreri $\quad .75$

Gentiana gentianella $\quad .75$

Gentiana kurroo $\quad .50$

Centiana prolata $\quad .75$

Gentiana purdomi $\quad .75$

Gentiana sino-ornata .35

Gentiana sino-ornata, very dark .35

Geranium cinereum $\quad .50$

Geranium lancastriense $\quad .30$

Crcranium pylzowianum $\quad .40$

Geranium sanguineum $\quad .25$

Geranium wallichianum $\quad .30$

Haberlea ferdinandi-coburgi $\quad 1.00$

Haberlea rhodopensis $\quad .80$

Hclianthemum tuberaria $\quad .35$

Horminum pyrenaicum $\quad .30$

Houstonia, Millard's varicty $\quad .50$

Hutchinsia alpina $\quad .25$

Hypericum coris $\quad .30$

Hypericum reptans $\quad .40$

Inula cnsifolia $\quad .30$

Iris arenaria $\quad .75$

Iris gracillipes $\quad .75$

Iris tricuspis $\quad .50$

Lcontopodium alpinum $\quad .25$

Lithospermum prostratum $\quad .50$

Lychnis lagascae $\quad .35$

Omphalodes cappadocica $\quad .30$ 
Omphalodes verma .35

Ononis rotundifolia $\quad .50$

Oxalis adenophylla $\quad .50$

Papaver alpinum $\quad .25$

Phyteuma scheuchzeri $\quad .25$

Potentilla nitida $\quad .75$

Pratia angulata 35

Primula anisodora .35

Primula auricula alpina $\quad .25$

Primula cashmiriana $\quad .25$

Primula crispa $\quad .35$

Primula farinosa $\quad .35$

Primula frondosa $\quad .25$

Primula glycosna $\quad .35$

Primula helenae $\quad .50$

Primula juliae $\quad .35$

Primula littoniana $\quad .60$

Primula marginata $\quad .75$

Primula minima $\quad 1.00$

Primula moerheimi .25

Primula mooreana $\quad .35$

Primula nivalis $\quad .75$

Primula pudibunda $\quad .35$

Primula rosea $\quad .40$

Primula spectabilis $\quad .75$

Primula veitchi $\quad .35$

Primula vincaeflora $\quad 1.00$

Primula waltoni $\quad .35$

Primula wilsoni $\quad .35$

Primula wulfeniana $\quad .75$

Prunclla grandiflora $\quad .20$

Ramodia nataliae $\quad 1.00$

Ramondia pyrenaica $\quad .80$

Saxifraga acizoon labradorica .3)

Saxifraga acizoon lutea $\quad .35$

Saxifraga acizoon notata $\quad .35$

Saxifraga acizoon rosea $\quad .35$

Saxifraga aeizoon rex $\quad .50$

Saxifraga aeizoon rosularis $\quad .35$

Saxifraga apiculata $\quad .35$

Saxifraga apiculata alba $\quad .30$

Saxifraga baldensis $\quad .50$

Saxifraga boryi $\quad .60$

Saxafraga burseriana magna $\quad .75$

Saxafraga burseriana minor $\quad 1.00$

Saxafraga burscriana sulphurea .75

Saxifraga cartilaginea $\quad .25$

Saxifraga cholentiana $\quad .50$

Saxifraga cochlearis $\quad .25$

Saxifraga Dr. Ramsey $\quad .50$ 
Saxifraga elizabethae

Saxifraga faldonside

Saxifraga ferdinandi-coburgi $\quad .40$

Saxifraga gaudini $\quad .35$

Saxifraga grisebachi $\quad 1.25$

Saxifraga haagi $\quad .50$

Saxifraga hosti $\quad .50$

Saxifraga irvingi $\quad 1.00$

Saxifraga juniperiana $\quad .50$

Saxifraga lagaveana $\quad .25$

Saxifraga lilacina $\quad 1.25$

Saxifraga lingulata alberti $\quad .35$

Saxifraga longifolia $\quad .40$

Saxifraga marginata $\quad .75$

Saxifraga media $\quad 1.00$

Saxifraga obristi $\quad .75$

Saxifraga oppositifolia $\quad 1.00$

Saxifraga paulinae $\quad 1.00$

Saxifraga pectinata $\quad \mathbf{3 5}$

Saxifraga petraschi $\quad 1.00$

Saxifraga sancta $\quad .50$

Saxifraga scardica $\quad .75$

Saxifraga stanborgi $\quad \mathbf{3 5}$

Saxifraga stenoglossa $\quad \mathbf{3 5}$

Silcne acaulis carminea $\quad .50$

Silene pudibunda $\quad .25$

Soldanella alpina $\quad \mathbf{3 5}$

Statice minuta $\mathbf{3 5}$

Thlaspi rotundifolium $\quad .50$

Thymus languginosus $\quad .25$

Thymus serpyllum albus $\quad .25$

Thymus serpyllum coccineus $\quad .25$

Trollius pumilus $\quad .40$

Veronica incana $\quad .30$

Veronica rupestris $\quad .30$

Vorcnica rupestris rosea $\quad .35$

Veronica saxatilis $\quad .25$

\section{Forcign Rock Shrubs}

Azalea obtusa $\quad .50$

Tiruckenthalia spiculifolia $\quad .75$

Calluna vulgaris nana $\quad .50$

Chamaecyparis minima glauca 2.00

Chamaecyparis nana $\quad .75$

Chamaecyparis obtusa

lycopodioides $\quad 2.50$

Cistus laurifolius $\quad .50$

Cistus salvifolius $\quad .50$ 
Cistus villosus $\quad .50$

Cotoneaster sp.? Jap. prostrate .75

Cotoneaster dammeri .50

Cotoneaster microphylla glacialis .75

Cotoneaster rotundifolia

$$
\begin{array}{ll}
\text { prostrata } & .75
\end{array}
$$

Cytisus ardoini $\quad .75$

Cytisus decumbens $\quad .50$

Cytisus kewensis $\quad .75$

Cytisus schipkaense $\quad .75$

Cryptomeria japonica nana $\quad .60$

Daphne cneorum $\quad .50$

Daphne mezereum $\quad .35-1.00$

Dryas sundermani $\quad .35$

Erica ciliaris $\quad .35$

Erica tetralix $\quad .50$

Genista dalmatica $\quad .75$

Genista saggitalis $\quad .50$

Globularia nana $\quad .35$

Hedera helix conglomerata $\quad .35$

Ilex crenata $\quad .50$

Jasminum parkeri $\quad 2.00$

Juniperus japonica aurea $\quad .75$

Pinus mugho $\quad .75$

Polygonum vaccinifolium $\quad .50$

Rhododend ron chryscum, 2 yr. 1.00

Rhododendron ferrugineum $\quad .50$

Rhododendron impeditum 2.25

Rhododendron neriiflorum, 2 yr. 1.50

Rhododendron racemosum .75-1.50

Rhododendron virgatum $1.50-2.50$

Rosa gloria mundi $\quad .50$

Rosa polyanthus nana multiflora .50

Rosa rouletti $\quad .50$

Spiraca bullata $\quad .75$

Thymus azoricus $\quad .25$

Vaccinium vitis-idaca $\quad .50$

Veronica gutheriana .40

Veronica hulkeana $\quad .60$

\section{Native Alpine Plants and Shrubs}

Anemone drummondi $\quad .35$

Anemone pulsatilla $\quad .35$

Boykinia jamesi $\quad .35$

Caltha Teptosepala $\quad .35$

Ceanothus prostratus, in pots $\quad .50$

Coptis asplenifolia $\quad .50$

Coptis occidentalis $\quad .35$ 
Cypripedium californicum $\quad .75$

Cypripedium montanum $\quad .50$

Delphinium nudicaule $\quad .35$

Douglasia lacvigata $\quad .50$

Draba nova-olympica $\quad .75$

Dryas drummondi .35

Dryas octopetala $\quad .35$

Galax aphylla $\quad .50$

Gaultheria ovatifolia $\quad .60$

Gaultheria procumbens $\quad .50$

Gormania watsoni .25

Hepatica tribola $\quad .50$

Houstonia coerulea $\quad .25$

Houstonia serpyllifolia $\quad .25$

Hydastylus borealis $\quad .50$

Hypericum scouleri $\quad .40$

Iris cristata $\quad .35$

Iris tenax $\quad .35$

Iris verna $\quad .35$

Jeffersonia diphylla $\quad .50$

Juniperus sibirica nana $\quad .75$

Juniperus horizontalis $\quad .75-1.50$

Leiophyllum prostratum $\quad .1 .25$

Lewisia columbiana rosea $\quad .30$

Lewisia tweedyi $\quad .75$

Lupinus lyalli $\quad .50$

Lutkea pectinata $\quad .35$

Mimulus lewisi

Mimulus primulnides $\quad .50$

Pentstemon barrettae $\quad .35-.75$

Pentstemon diffusus $\quad .35$

Pentstemon menziesi $\quad .50$

Pentstemon menziesi davidsoni .35

Pentstemon pubescens $\quad .35$

Pentstemon rattani-minor $\quad .25$

Pentstemon rupicola $\quad .50$

Phlox divaricata $\quad .25$

Phlox verna $\quad .25$

Polemonium clegans $\quad .25$

Polemonium carneum $\quad .50$

Potentilla tridentata $\quad .25$

Primula parryi $\quad .50$

Rhodiola rosea $\quad .20$

Romanzoffia sitchensis $\quad .50$

Romanzoffia unalaschensis $\quad .50$

Saxifraga adscendens $\quad .50$

Shortia galacifolia $\quad .50$

Silene acaulis $\quad .35$

Sisyrinchium, blue $\quad .25$

Sisyrinchium grandiflorum $\quad .35$ 
Sisyrinchium inflatum $\quad .50$

Sisyrinchium inflatum album $\quad .75$

Spiraea caespitosa $\quad 1.00$

Spiraea hendersoni $\quad 1.00$

Stylophorum diphyllum $\quad .35$

Vancouveria hexandra $\quad .25$

$\begin{array}{ll}V \text { iola bellidifolia } & .40\end{array}$

Viola cucullata $\quad .30$

Viola pedata $\quad .35$

Viola pedata bicolor $\quad .40$

$\begin{array}{ll}V \text { iola primulifolia } & .40\end{array}$

Native Rock Ferns

Asplenium platyneuron $\quad .50$

Asplenium trichomanes $\quad .35$

Botrychium silaifolium $\quad .50$

Cheilanthes gracillima $\quad .50$

Cryptogramma acrostichoides $\quad .35$

Pellaea brachyptera $\quad .75$

Pellaca bridgesi $\quad .75$

Pellaea densa $\quad .35$

Phegopteris phegopteris $\quad .50$

Polystichum viviparum (India) .50

Postagc or express on planes is charged to the purchaser.

\section{JULIUS ANTHON}

2215 East 46th Street

Seattle, Washington U. S. A .

Phone Kenwood 4939 